\title{
Le Feu et son Double
}

\section{Eunice Dutra Galéry}

«Et puis, quoi encore? - l'ambiguité, à quoi, de par sa nature, la parole est vouée.»

JACQUES LACAN

\section{FEU FOU}

Feu désert

feu des nuits sans même une eau limpide

dans le blanc de l'oeil

feu de paille et de boue

feu stérile

feu pourri

nous aurions pu te détruire

te faire rendre ta dernière étincelle

te prendre à la gorge et t'étouffer

t'éteindre

ou te disperser en plusieurs foyers

que nous aurions ensuite foulés du pied

mais nous avions besoin de ta chaleur

et de ta lueur

pour habiter nos ombres glaciales

la poudre d'encre dans nos veines nous affaiblissait

et par-dessus tout nous pesait cette obscurité

dans laquelle il nous fallait regrouper notre être

sans cesse écartelé aux quatre vents de l'opaque

et toi

feu idiot

tu brûlais tout sans voir et sans savoir

OBSERVATION: Toutes les citations de FEU FOU et des autres poèmes de Giguère qui n'ont pas été indiquées ont été prises dans L'Age de la parole. Ottawa, Ed. de l'Hexagone, 1965. 
nos pires erreurs et nos lettres d'espoir

tu nous brûlais les paupières

tu nous brûlais la poitrine

nos châteaux de cartes et nos ignobles ruines

feu idiot

feu fou

tu te jouais de nous

mais dans ton ombre déjà nous préparions ta cendre.

(ROLAND GIGUËRE - L'Age de la parole)

Toute une longue tradition occidentale voit dans le feu l'éclair divin volé aux dieux, l'élément de purification à travers lequel les offrandes arrivent aux divinités, l'élément béni où Yahweh descendait pour parler aux hommes:*

"Le Seigneur lui apparut dans une flamme qui jaillissait au milieu d'un buisson. Moïse le regardait: le buisson flambait mais ne se consumait pas."

(L'Exode, 3:2)

Le feu est aussi le symbole de l'alliance et de la protection divines, de la sagesse et de la faveur du dieu:

"Alors ils virent paraître comme des langues de feu qui, se partageant, vinrent se poser sur chacun d'eux. Ils furent tous remplis du Saint-Esprit, et se mirent à parler des langues étrangères selon que l'Esprit leur donnait de s'exprimer."

(Actes des Apôtre, 2:3,4)

et, dans un élargissement du symbole, le feu représente les sentiments les plus élevés, la "flamme" classique:

* Rien que pour nous tenir à la tradition occidentale. Les mythes du feu chez les indiens de l'Amérique viennent renforcer l'idée. Cf. LévY-STRAUSS,
Le Cru et le Cuit. 
"Ce n'est pas qu'en effet contre mon père et moi Ma flamme assez longtemps n'ait combattu pour to $i^{\text {" }}$

(Corneille, LE CID)

où, à l'âge du baroque, ce "fogo que arde sem se ver", dont parle Camões dans ses sonnets.

Cette mythologie du feu se conserve à travers les temps jusqu'à nos jours, toujours comme quelque chose qui vient des hauteurs:

"........ Le feu, des cirques pur descend

$I l$ change le mont chauve en auguste trophée"

(Paul Valéry, Guvres)

Ce caractère sacré du feu renferme aussi un des ses aspects, et non des moins importants: le feu est parole, ou bien l'élément par lequel la parole est donnée aux hommes, aussi bien dans la Bible - les "langues de feu" qui descendent sur les apôtres et leur donnent la maîtrise des langues étrangères - que chez Valéry, où la parole est parole d'Orphée, qui change et recrée le monde.

Pourtant ce même feu, objet d'adoration, est aussi objet de crainte. Le feu purificateur est aussi le feu infernal, le feu du châtiment, le feu qui pleut sur Sodome, la foudre de Zeus, le feu du martyre et de l'Inquisition, celui qui détruit la nature, comme chez Castro Alves, "a queimada é uma serpente", image que nous retrouvons chez Giguère, "les jours où le feu rampait sous la cendre" (ROSES ET RONCES), ou bien encore celui qui détruit la culture, comme dans FAREINHEIT 478 le feu qui dévore, qui déforme, que les hommes ne peuvent pas dompter, jusqu'au monstrueux champignon qui un jour a éclos sur Hiroshima, le feu aveugle qui devient l'image même de la mort.

Ce caractère paradoxal de la relation de l'homme au feu est repris dans le poème de Giguère, FEU FOU, où un élément nouveau apparaît: la désacralisation et la dégradation du feu.

Attirance et répulsion, les deux pôles qui créent la tension du poème, et dont nous examinerons les divers aspects: d'abord sur le plan sonore, où les phonèmes antérieurs, $/ \mathrm{F} /, / \mathrm{V} /$, ou bien $/ \mathrm{T} /$ et $/ D /$ ont nettement le dessus et font l'alternance presque exclusive 
avec les $/ R_{4} /$, sorte de roulement sourd qui accompagne l'extrême tension des lèvres dans la prononciation des mots.

L'onomatopée représentée par cette alternance nous rappelle le bruit du feu aussi bien que celui du vent; les consonnes plosives, dites "dures", comme $/ \mathrm{P} /, / \mathrm{T} /$ ou $/ \mathrm{D} /$, qui s'accumulent dans des vers tels que

\title{
"nous aurions pu te détruire \\ te faire rendre ta dernière étincelle"
}

font écho à l'agressivité des mots.

Les consonnes longues, sifflantes, telles que $/ \mathrm{V}_{i} /, / \mathrm{S} /$ et surtout $/ F /$, rappellent une certaine lassitude, le désespoir qui rallonge les mots et l'incertitude entre deux attitudes opposées - comme quelqu'un qui s'arrête un instant pendant une course, pour reprendre le souffle.

Ce partage de l'être est déjà indique dans le titre, F'EU FOU, deux mots brefs où la répétition du $/ \mathrm{F} /$ suivi de $/ \varnothing /$ et de $/ \mathrm{U} /$ mime le mouvement de la bouche qui souffle, dévoile le désir et l'ambiguité des rapports qui relient l'homme au feu, car le souffle sert aussi bien à éteindre qu'à rallumer le feu. Ce souffle ambigu charrie tout le poème, dans un système d'oppositions/similitudes qui se révèle dans tous les autres niveaux: dans l'emploi des temps et des modes verbaux nous remarquons que toutes les actions de l'homme sont au conditionnel passé, mode de l'hypothétique non-réalisé:

\section{"nous aurions foulés"}

ou bien sont introduites par ce même conditionnel passé, modalisé en plus par le verbe pouvoir:

\author{
"nous aurions pu \\ te détruire \\ te faire rendre ta dernière étincelle \\ te prendre à la gorge \\ t'étouffer \\ t'éteindre \\ te disperser
}


L'emploi du conditionnel passé ici indique déjà cet

"Effet de rétroversion par quoi le sujet à chaque étape devient ce qu'il était comme d'avance et ne s'annonce: il aura été qu’au futur antérieur." 1

puisque l'emploi du futur antérieur, dans un pareil cas, est très proche de celui du conditionnel passé, indiquant une attitude de locution - l'insinuation. Dans le poème, l'insinuation est une menace voilée qui ne se réalise pas.

Les verbes à l'imparfait, au contraire, servent à décrire soit un état où se trouve l'homme, révélant l'impuissance et la faiblesse:

nous avions besoin
nous affaiblissait
nous pesait
il nous fallait regrouper;

soit une promesse, plus que douteuse, d'action de l'homme:

nous préparions ta cendre;

soit l'action du feu, le seul qui en est vraiment capable:

tu brûlais

tu te jouais.

Sur le plan lexical, outre les expressions verbales indiquées plus haut, nous voyons trois isotopies dominantes se dégager (v. table I, annexe) : celle du feu, celle de l'homme et celle de la dégradation.

C'est par la médiation de la dégradation que l'homme va essayer de rendre le feu son égal. II lui rajoute des épithètes comme "fou", "idiot", "désert", "stérile", en projetant sur lui et sa misère et ses intentions, car, en le rendant son égal, l'homme a l'illusion de pouvoir le dompter, le rendre son esclave. Pourtant cet esclave est plus puissant que son maître et nous voyons, déjà à ce niveau, s'ébaucher

1. LACAN, Jacques. Ecrits. Paris, Seuil, 1966, pp. 808-809. 
la relation Maître-Esclave dont parle Hegel, aussi bien que l'inversion des rôles présente dans tout rapport intersubjectif, puisque

\begin{abstract}
"tout sujet est pareillement constitué par un Autre, tout sujet est Esclave d'un mấtre qui est l'Autre, fût-il la Mort. Aussi bien, le prétendu Man̂tre est-il dans l'intersubjectivité reconnue, l'Esclave de l'Esclave, son signifié" 2
\end{abstract}

Le feu devenu personnage par ce procès d'humanisation devient le signifiant premier dont l'homme est le signifié: à "feu" nous pourrions substituer "homme" et dire:

Homme fou

Homme désert

Homme sans même une eau limpide... et ainsi

de suite, pour bien montrer le caractère interchangeable de ces deux apparents contraires - pour arriver au démasquage de cette opposition qui devient dédoublement, le rapport essentiel du poème, celui de l'homme à l'homme.

L'identité entre l'homme et le feu se fait encore percevoir dans d'autres indices lexicaux: ainsi, si l'homme sent peser sur lui l'obscurité, s'il se meut dans "l'opaque" et dans les ombres, le feu, lui, est un "feu des nuits", qui ne répand pas de lumière, mais juste une "Iueur" - dégradation de la lumière - il n'est pas limpide (sans même une eau limpide), il est "de boue"; ainsi, au lieu de s'opposer, les mots se rejoignent dans une même signification: "l'opaque" par rapport à l'homme, est "sans limpidité" par rapport au feu; les "ombres", "l'ombre", deviennent "lueur". La seule caractéristique du feu qui échappe à cette identité est la chaleur; remarquons pourtant que cette chaleur dont l'homme a besoin, se nourrit de l'homme et de son oeuvre:

2. KREMER-MARIETTI, A. Lacan et la rhétorique de l'inconscient. Paris, Aubier-Montaigne, 1978, pp. 113-114. 
"tu brûlais (...)

nos pires erreurs et nos lettres d'espoir

tu nous brûlais les paupières

tu nous brûlais la poitrine

nos châteaux de cartes et nos ignobles ruines"

L'homme a besoin de la chaleur pour survivre - mais cette chaleur a son origine dans la combustion de l'homme même qui, au contraire du phoenix de la fable, brûle et se consume pour ne pas revenir. Et ici, l'image nous vient irrésistiblement à l'esprit, du serpent qui mord sa queue... Car enfin, qu'est-ce que la vie, sinon la combustion permanente - par l'oxygène, par le souffle qui est, en même temps, respiration et parole? "Au principe était le Verbe..." et nous retombons dans l'imagerie biblique:

"Le Seigneur Dieu forma l'homme avec la poussière du sol et lui inspira dans la narine un souffle de vie et l'homme devint un être vivant."

(Génèse, 2:7)

Souffle - parole et vie - le premier aussi fugace que les deux autres, nous passons naturellement au "feu de paille" qui s'enflamme vite et s'éteint encore plus vite, qui rejoint le "feu stérile", car il ne dure pas assez pour répandre chaleur et lumière, feu fugace par où on va au "feu de boue" et au "feu pourri" - le feu follet, doublement rappelé: sur le plan étymologique - fou-fol-follet — et sur le plan physique - manque de chaleur et de lumière - juste une lueur. Encore ici l'imagerie biblique revient: l'homme est fait de la "poussière du sol", mais le sol était imbibé d'eau" de la boue, donc, mais de la boue, elle aussi, atomisée: poussière et vapeur.

Le feu follet est une

"flamme légère et fugitive (...) qui se dégage des endroits marécageux et des lieux, tels les cimetières, où des matières animales se décomposent." 3

* «mais de la terre montait une vapeur qui en arrosait toute la surface; le Seigneur Dieu forma l'homme avec la poussière du sol» (Génèse, $2: 6,7$ )

3. Dictionnaire Nouveau Petit Larousse. Paris, Larousse, 1966, 113e éd. 
Le feu, dans le poème, se nourrit de l'homme; le feu follet se dégage de la décomposition des matières animales et le langage courant utilise les mots "cendres" et "feu" dans les nécrologes feu, qui, à partir d'une double origine, focus et fatutus est abouti à la même graphie, deux mots, deux sens, et l'idée de la mort derrière; le feu et la cendre sont donc liés à l'idée de la mort; de même, l'ombre $\left(\sigma x^{\prime} \alpha\right)$, celui qui a vécu. Cette lourde charge connotative de mort pèse sur le dernier vers du poème:

"mais dans ton ombre déjà nous préparions ta cendre."

Décomposition, dégradation, l'identité se poursuit sous d'autres aspects: le feu et le sang ont la même couleur, qui n'est pas nommée dans le poème - et pour cause: ce feu des nuits, à qui on prépare dans l'ombre la cendre, dont la lumière est dégradée en lueur, a la même coloration de la poudre d'encre qui remplit les veines de l'homme. Ce feu et ce sang sont, tout comme le ciel et la mer du poème de Baudelaire, "noirs comme de l'encre".

Et encore ici, tout est atomisé: le feu devient cendre, poudre qui se disperse, elle aussi, "aux quatre vents de l'opaque", mais "les cendres aident le vent à retourner chez lui" ${ }^{4}$ et le sang qui aurait pu être confondu avec la boue - liquide et visqueux - devient "poudre d'encre" - celle qui enlève la force et qui nous fait penser encore à un autre poème de Giguère:

"Il y a longtemps que je ne vous ai écrit. À force de vouloir tout dire, je ne sais plus que dire." 5

L'identification de l'homme au feu se fait remarquer aussi sur le plan des rapports de personnes; l'opposition JE/TU frappe le lecteur au premier abord. Entre JE et TU, monologue de JE pluriel dirigé à TU singulier, l'homme enviant au feu son unité, opposée à la fragmentation de l'homme - le JE devenu NOUS, un NOUS

4. GIGUERE, Roland. Frorêt vierge folle. Ottawa, Ed. de l'Hexagone, 1978, «Un jour ou l'autre», p. 46.

5. GIGUERE, Roland. La Main au feu. Ottawa, Ed. de l'Hexagone, 1973 ; «Lettres à l'évadé», p. 43 . 
qui ne peut pas faire illusion, puisqu'il n'est pas une marque de solidarité, mais celle de la dispersion de l'homme, qui doit toujours

$$
\begin{aligned}
& \text { "............... regrouper son être } \\
& \text { sans cesse écartelé aux quatre vents de l'opaque" }
\end{aligned}
$$

Mais, pour évidente qu'elle soit à première vue, cette opposition NOUS/TU renferme, elle aussi, son ambiguité: l'unité que l'homme envie au feu, n'est-elle pas sa propre unité dont l'espoir est à jamais perdu? Désespérant d'être l'égal du feu, l'homme essaie de le rendre son égal: soit par lui prêter des caractéristiques humaines:

FEU $\left\{\begin{array}{l}\text { blane de l'oeil } \\ \text { gorge } \\ \text { idiot } \\ \text { fou }\end{array}\right.$

soit par le renouvellement d'expressions habituellement appliquées à l'homme, telles que "une larme au coin de l'oeil", qui devient "une eau limpide dans le blanc de l'oeil", ou bien "rendre le dernier soupir" qui se transforme en "rendre ta dernière étincelle", soit par prêter au feu des intentions dont l'homme seul est capable: "tu te jouais de nous".

L'homme voit dans le feu à la fois son semblable et son ennemi - ce feu humanisé est le reflet même de l'homme, son moi, qui devrait être indivisible et son Autre qui l'atomise.

L'idée de la mort est présente encore ici, si nous considérons que cette identification de l'homme au feu correspond à la fase narcissique, au stade du miroir et que

"Ce noeud imaginaire absolument essentiel", le narcissisme, détient le rapport de l'imago à l'instinct de mort" ${ }^{6}$

Le rapport dialectique qui s'établit, attirance et répulsion, besoin et désir de destruction, est celui qui s'établit entre le maître et l'esclave: deux entités interdépendantes, qui ne peuvent pas subsister

6. KREMER-MARIETTI, A. Op. cit., p. 92 . 
l'une sans l'autre, et qui se haïssent à cause même de leur interdépendance, des chaînes qui les lient.

Les significations qui se dégagent de la lecture du poème, soient l'agressivité, les rapports maître-esclave et la fragmentation/ atomisation qui récèle le désir d'unité nous mènent à une nouvelle voie dans le déchiffrage de ses significations multiples: la mort et le "corps morcelé" dont parle Lacan, concepts, eux aussi, étroitement liés.

Le "corps morcelé", explicité dans les vers ".... il nous fallait regrouper notre être sans cesse écarteté..."

est présent dans toute une nouvelle isotopie, celle de l'atomisation:

paille

boue

disperser

plusieurs

poudre

écartelé

cendre.

Selon Lacan, au premier moment du "stade du miroir", quand l'enfant voit son image et la voit comme l'Autre, il ressent l'angoisse de son corps morcelé:

'l'unité du corps, loin d'être donnée et première, s'acquiert par des péripéties angoissantes à partir de la vision d’un corps morcelé (d'où: angoisse du corps morcelé)" 7

L'Autre, le double dans le miroir, est une unité, tandis que lui, qui ne se reconnaît pas encore dans ce double, qui voit son propre corps par parties, se trouve "écartelé" - d'où l'agression envers ce double qu'il envie, puisque

"Quoi qu'il en soit ce que le sujet trouve en cette image altérée de son corps, c'est le paradigme de toutes les formes

7. KREMER-MARIETTI, A. Op. cit., p. 87 . 
de la ressemblance qui vont porter sur le monde des objets une teinte d'hostilité en y projetant l'avatar narcissique, qui, de l'effet jubilatoire de sa rencontre au miroir, devient dans l'affrontement au semblable le déversoir de la plus intime agressivité." 8

Ce double qui hante l'homme, est annoncé dès le titre du poème: FEU FOU, où un seul graphème fait la différence - et encore faut-il penser que FEU s'écrivait FOU jusqu'au commencement du XI. ${ }^{e}$ siècle; ${ }^{*}$ nous aurons donc un titre tautologique: FOU FOU, aux termes interchangeables, le moi et son double, le feu qui est l'homme, le fou qui est l'homme, doublement feu on doublement fou.

Depuis les études de Freud dans L'Inquiétante Etrangeté nous savons que la hantise du double rejoint la hantise de la mort. Et quand l'homme s'en prend au feu, ce feu fou, voilà que "ce n'est rien que le kakon de son propre être, que l'aliéné cherche à atteindre dans l'objet qu'il frappe." 9

L'agressivité envers l'autre devient l'agressivité contre soi-même, jusqu'au suicide, et nous plongeons dans le domine de l'imaginaire lacanien, domaine "dont le pivot est une relation intersubjective mortelle", ${ }^{10}$ caractéristique de la relation hegelienne du maître et de l'esclave.

Selon Lacan, l'esclave attend la mort du maitre, car cette mort s'interpose entre lui-même et la mort; idée qui renforce la signification du dernier vers du poème de Giguère, l'action de l'homme annoncée, non plus au conditionnel passé, temps de l'hipothétique non-réalisé, mais à l'imparfait:

Il faut encore noter que tout le poème est raconté au passé; on peut alors supposer que celui qui raconte a survécu, le temps de

"mais dans ton ombre déjà nous préparions ta cendre"

8. LACAN, Jacques. Op. cit., pp. 808-809.

* DAUZAT, Albert. Dictionnaire Etymologique.: I. feu, subst. (fou, Xe. s., Eulalie, 1100, Roland;...) GRANDSAIGNES D'HAUTERIVE, $R$. Dictionnaire d'ancien français. FOU I - une des graphies de FEU; Les merveillus tempez, $\mathrm{E}$ fous e flambes. (XIe. s., Rol.)

9. LACAN, Jacques. Op. cit., p. $\mathbf{1 7 5}$.

10. LACAN, Jacques, Le Séminaire, Libre I. Paris, Seuil, 1975, pp. 248-249. 
l'énonciation étant postérieur au temps de l'énoncé, tout en tenant compte que

"Je est sujet de l'énonciation, je l'est de l'énoncé. L'un énonce le souvenir qui est rapporté à l'autre comme à celui qui a vécu l'aventure, et il paraît qu'ils sont le(s) même(s). Mais le lecteur est bien vite confronté à un troisième locuteur, qui précède Je d'un cran et qui est censé déclarer à l'orée du livre: "JE vais vous raconter une histoire, puisque ceci s'intitule roman"; ce JE marque la limite du texte, qui le sépare du monde et le relie au monde." 11

Pourrait-on conclure que l'homme sort vainqueur de cette lutte pour une identité et qu'il se retrouve enfin UN par l'anéantissement de son maître, ce maître qui se réalise par la médiation du travail de l'esclave, dont il se nourrit, comme nous l'avons montré plus haut?

Revenons à Lacan:

"Quand le maître sera mort, tout commencera (...). Il a raison d'ailleurs, l'esclave, c'est à juste titre qu'il peut jouer sur cette attente. Pour reprendre un mot qu'on attribue à Tristan Bernard, au jour où on l'arrêtait pour l'emmener dans le camp de Dantzig - Jusqu'ici nous avons vécu dans l'angoisse, maintenant nous allons vivre dans l'espoir.

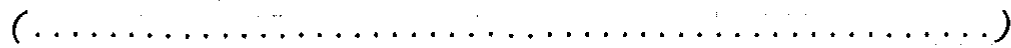
l'esclave a beaucoup à attendre de la mort du mâ̂tre. Au-delà de la mort du mâ̂tre, il faudra bien qu'il s'affronte à la mort, comme tout être pleinement réalisé, et qu'il assume, au sens heideggerien, son être-pour-la-mort." 12

Image du corps morcelé confrontée à l'unité du double, condition déterminante qui établit la lutte mortelle entre le je et son double, entre l'Esclave et le Maître, lutte qui ne peut aboutir qu'à la Mort - hantise dont tout le poème est impregné et qui reflète l'ambivalence essentielle de l'homme, partagé entre Eros et Thanatos, la pulsion de vie et la pulsion de mort.

11. BELLEMIN-NOËL, Jean. Vers linconscient du texte. Paris, PUF, 1979 , p. 197.

12. LACAN, Jacques, Le Séminaire, Livre I. p. 315. 


\begin{tabular}{|c|c|c|c|c|c|c|c|}
\hline \multicolumn{6}{|c|}{ ISOTOPIES INDICATIVES } & \multicolumn{2}{|c|}{ ISOTOPIES SUBJONCTIVES } \\
\hline & FEU & OMBRE & HUMAIN & LIQUIDE & ATR & DEGRADATION & ATOMISATION \\
\hline $\begin{array}{c}\text { Titre } \\
1 \\
2 \\
3 \\
4 \\
5 \\
6 \\
7 \\
8 \\
9 \\
10 \\
11 \\
12 \\
13 \\
14 \\
15 \\
16 \\
17 \\
18 \\
19 \\
20 \\
21 \\
22 \\
23 \\
24 \\
25 \\
26 \\
27 \\
28 \\
29 \\
30\end{array}$ & $\begin{array}{l}\text { feu } \\
\text { feu } \\
\text { feu } \\
\text { feu } \\
\text { feu } \\
\text { feu } \\
\text { te } \\
\text { te/ta/étincelle } \\
\text { te/t' } \\
\text { t' } \\
\text { fovers } \\
\text { ta/chaleur } \\
\text { ta/lueur } \\
\\
\\
\\
\text { to } \\
\text { feu } \\
\text { tu/brûlais } \\
\text { tu/brûlais } \\
\text { tu/brûlais } \\
\text { feu } \\
\text { feu } \\
\text { tûte } \\
\text { ton/ta/cendre }\end{array}$ & $\begin{array}{l}\text { luits } \\
\text { steindre } \\
\text { ombres } \\
\text { encre } \\
\text { obscurité } \\
\text { opaque }\end{array}$ & $\begin{array}{l}\text { fou } \\
\text { blane de l'oeil } \\
\text { nous/aurions pu } \\
\text { gorge } \\
\text { nous/pied } \\
\text { nous } \\
\text { nos } \\
\text { nous/nos/veines } \\
\text { nous } \\
\text { nous/notre } \\
\\
\text { voir/savoir } \\
\text { nos/espoirs/erreurs } \\
\text { nous/paupières } \\
\text { nous/poitrine } \\
\text { nos (2) } \\
\text { idiot } \\
\text { fou } \\
\text { nous } \\
\text { nous }\end{array}$ & \begin{tabular}{|l|} 
eau \\
boue \\
\\
\\
glaciales \\
encre
\end{tabular} & étouffer & \begin{tabular}{|c|} 
fou \\
désert \\
sans \\
paille/boue \\
stérile \\
pourri \\
détruire \\
dernière/étincelle \\
étouffer \\
éteindre \\
plusieurs \\
foulés \\
lueur \\
glaciales \\
poudre/affaiblissait \\
\\
écartelé/sans \\
\\
sans (2) \\
pires erreurs \\
brûlais \\
brûlais \\
idiot \\
fou \\
jouais \\
cendre
\end{tabular} & $\begin{array}{l}\text { paille/boue } \\
\text { étincelle } \\
\text { plusieurs }\end{array}$ \\
\hline
\end{tabular}




\section{BIBLIOGRAPHIE}

BELLEMIN-NOËL, Jean. Vers linconscient du texte. Paris, PUF, 1979.

CAMŌES, Luiz de. Sonetos. Rio, Ed. Agir, 1957.

CORNEILE, Pierre. Le Cid. Paris, Bordas, 1970.

DAUZAT, A. Dictionnaire Etymologique. Paris, Larousse, 1949, 10e éd.

DUBOIS, J. et alii. Retórica da poesia. Trad. de Carlos Felipe Moisés. S. Paulo, Cultrix/Edusp, 1980.

FAGES, J.B. Comprendre Jacques Lacan. Paris, Pensée/Privat, 1979.

FELMAN, S. La folie et la chose litteraire. Paris, Seuil, 1978.

FREUD, S. Obras completas. 3 t. Madrid, Biblioteca Nueva, 1973.

GIGUERE, Roland. La Main au feu. Ottawa, Hexagone, 1973.

- Foret vierge folle. Ottawa, Hexagone, 1978.

- L'Age de la parole. Ottawa, Hexagone, 1965.

GRANDSAIGNES D'HAUTERIVE, R. Dictionnaire d'Ancien Français. Paris, Larousse, 1947.

KREMER-MARIETTI, A. Lacan et la rhetorique de linconscient. Paris, Aubier-Montaigne, 1978.

KRISTEVA, J. et alii. Folle verite. Paris, Seuil, 1979.

LACAN, Jacques. Ecrits. Paris, Seuil, 1966.

- Le Seminaire, Livre I. Paris, Seuil, 1975.

. Le Seminaire, Livre II. Paris, Seuil, 1978.

. Le Seminaire, Livre XI. Paris, Seuil, 1973.

LEVIY-STRAUSS, C. Le cru et le cuit. Paris, Plon, 1964.

LAPLANCHE, J. et PONTALIS, J-B. Vocabulaire de la psychanalyse. Paris, PUF, 1978, 6e éd.

La Sainte Bible. Braine-le-Comte (Belgique), Ed. Maredsous, 1950.

MILNER, Max. F'reud et l'interpretation de la litterature. Paris, CDU/SEDES,

ROUDINESCO, E. L'inconscient et ses lettres. Paris, Maison Mame, 1975.

VALERY, Paul. Oeuvres. Paris, NRF, Bibl. de la Pléiade, 1950. 